\title{
Linguistische kontrastive Studien Deutsch-Thailändisch: Eine Bestandsaufnahme
}

\author{
Korakoch Attaviriyanupap (Nakhon Pathom)
}

\begin{abstract}
This article is a survey and synthesis of German-Thai linguistic contrastive studies conducted between 1978 and 2008. Its objective is to give an overview of linguistic contrastive studies on these two languages. The first part is the analysis and synthesis of all these Thai-German linguistic contrastive studies in terms of quantity, characteristics and researched topics as well as the methodology used. In the second part, all relevant factors leading to this resulted overall picture are discussed and suggestions for further development in this field of study are made.
\end{abstract}

\section{$1 \quad$ Einleitung}

Obwohl in Thailand seit mehr als 80 Jahren Deutsch unterrichtet wird, ${ }^{1}$ sind bisher nur wenige thai-deutsche linguistische kontrastive Untersuchungen erschienen. In einigen Arbeiten werden einzelne Ergebnisse dieser Untersuchungen zwar erwähnt (vgl. z. B. Saengaramruang 2007: 359f.), es fehlt aber ein systematischer Überblick darüber, welche Themenbereiche im Bereich der kontrastiven Linguistik bisher untersucht wurden. Dies hängt vor allem damit zusammen, dass sich kontrastive germanistische Arbeiten bisher nahezu ausschließlich der Entwicklung des Deutschunterrichts und des Germanistikstudiums in Thailand, beispielsweise der Geschichte, dem Curriculum, der entwickelten Lehrmethoden oder dem Lehrmaterial, der Zahl und Einstellung der Deutschlehrenden wie auch -lernenden etc., widmeten.

Der vorliegende Aufsatz berichtet von den ersten Ergebnissen aus dem anlaufenden Forschungsprojekt "Deutsche Grammatik aus der Perspektive des Thailändischen"2 und versucht die Forschungslage in Form einer Synthese darzustellen, indem Stärken und Schwächen der bereits durchgeführten linguistischen kontrastiven Studien Deutsch-Thailändisch diskutiert werden. Diese Synthese soll als Grundlage für die zukünftig durchzuführenden kontrastiven Untersuchungen sowie für fruchtbareren wissenschaftlichen Austausch dienen, indem die Faktoren, die zu dem hier gewonnenen Gesamtbild führen, zur Diskussion gestellt werden.

Kontrastive Ansätze lassen sich naturgemäß nicht nur in kontrastiven Studien finden. Die Beschreibung der Grammatik einer Sprache durch einen Nichtmuttersprachler kommt nicht selten unweigerlich einem Sprachvergleich von zwei Sprachsystemen gleich. Für die vorliegende Synthese wurden allerdings nur Arbeiten berücksichtigt, die sich explizit dem Vergleich der beiden Sprachen, Deutsch und Thailändisch, widmen, egal ob die ganze Arbeit oder nur bestimmte Teile davon kontrastiv ausgerichtet sind. Die hier besprochenen Arbeiten beschreiben nicht nur die Grammatik einer der beiden Sprachen (nur Deutsch oder nur Thailändisch), sondern stellen die beiden Sprachen einander gegenüber. Der Vergleich kann aber

\footnotetext{
1 Am Anfang wurde Deutsch nur in einigen Schulen als 2. Fremdsprache unterrichtet. Erst vor etwa 50 Jahren wurde Deutsch zum ersten Mal als Studienfach im Rahmen eines Bachelorstudiums angeboten.

2 Das Projekt wird vom Thailand Research Fund unterstützt.
} 
in Form einer eigenständigen linguistischen kontrastiven Analyse, ein Teil einer empirischen Untersuchung mit relevanten Aspekten oder aber auch ein wissenschaftlicher Aufsatz sein, der die Besonderheiten der beiden Sprachen zur Diskussion stellt. Behandelt werden linguistische kontrastive Studien Deutsch-Thailändisch, die zwischen 1978 und 2008 durchgeführt worden sind.

\section{2 Überblick über die linguistischen kontrastiven Studien von 1978-2008}

\subsection{Quantitative Daten}

Eine Bestandsaufnahme von Monographien wie auch von Aufsätzen, die sich inhaltlich mit der Grammatik des Deutschen und der des Thailändischen befassen, ergibt, dass bisher insgesamt 29 Arbeiten erschienen sind, die den in der Einleitung erwähnten Auswahlkriterien entsprechen. Da einige dieser Arbeiten das Ergebnis ein und derselben Untersuchung sind und dieselben Erkenntnisse vorlegen, ist im Hinblick auf den Inhalt von nur 20 Studien zu sprechen. Die folgende Tabelle zeigt die Verteilung der Arbeiten, die im Folgenden im Detail besprochen werden.

\begin{tabular}{|c|c|c|c|c|c|}
\hline & & $\begin{array}{c}1978- \\
1987\end{array}$ & $\begin{array}{c}1988- \\
1997\end{array}$ & $\begin{array}{l}1998- \\
2008\end{array}$ & insgesamt \\
\hline Monogra & ien & 2 & 1 & 12 & 15 \\
\hline Aufsätze & zu anderen Forschungsthemen & 0 & 1 & 4 & 5 \\
\hline & $\begin{array}{l}\text { mit denselben Inhalten wie in den } \\
\text { Monographien }\end{array}$ & 0 & 1 & 8 & 9 \\
\hline Anzahl d & schriftlichen Arbeiten zu kontrastiv & & & & 29 \\
\hline Anzahl d & behandelten Studien & & & & 20 \\
\hline
\end{tabular}

Anzahl der linguistischen kontrastiven Studien Deutsch-Thailändisch

Die Monographien sind größtenteils im Rahmen von Master- oder Magisterstudiengängen oder von Promotionen durchgeführt worden: Von den 15 Monographien liegen 9 Masterbzw. Magisterarbeiten und 3 Dissertationen vor. Dazu kommen noch 3 selbstständige Forschungsprojekte. Eine Habilitationsschrift, die einzige Schrift dieses Typus, stellt eine Ausnahme in der binären Kategorisierung (studiumsgebunden oder selbstständig) dar. Mit der Habilitation erwirbt man im deutschsprachigen Raum einen akademischen Titel, der im thailändischen akademischen System keine Entsprechung findet und aus thailändischer Sicht als ein selbstständiges Forschungsprojekt gilt. Bis auf eine Magisterarbeit (Namsoongnein 1987) wurden alle anderen Masterarbeiten ${ }^{3}$ im Rahmen eines Studiums in Thailand verfasst, während alle Dissertationen an deutschsprachigen Universitäten angenommen wurden. Auch unter den Aufsätzen gibt es nur einen Artikel, der im deutschsprachigen Raum publiziert wurde (vgl. Attaviriyanupap 2006).

Aus den obigen Daten ist ersichtlich, dass im Bereich der kontrastiven Analysen der deutschen und der thailändischen Sprache noch beträchtlicher Bedarf besteht, zumal kontrastive Studien über dieses Sprachpaar erst in den letzten 10 Jahren durchgeführt wurden. Alle oben aufgeführten Arbeiten wurden von thailändischen Germanisten verfasst, und zwar zum großen Teil in Thailand. Arbeiten in der Gegenrichtung, nämlich thai-deutsche linguistische kontrastive Studien von deutschen Thaiisten, die den in der Einleitung angeführten Kriterien entsprechen, sind bisher keine erschienen. Dies ist auf die Tatsache zurückzuführen, dass sich

\footnotetext{
3 Das thailändische Hochschulsystem orientierte sich von Anfang an am amerikanischen System. Das Erststudium im traditionellen deutschen Sinne mit einem Magisterabschluss entspricht deshalb den zwei einander folgenden Stufen Bachelor- und Masterstudium in Thailand. Im Folgenden werden die beiden Begriffe, "Master" und "Magister" (bzw. "Master-" und "Magisterarbeit") verwendet, um genau zu beschreiben, um welche Art von Studiengang es sich jeweils gerade handelt.
} 
deutsche Thaiisten bis jetzt mehrheitlich Themen im Bereich der Literatur, Kunst, Kultur und Geschichte gewidmet haben. Wenn man aber nur von grammatischer Beschreibung des Thailändischen aus deutscher Sicht spricht, liegen einige relevante Arbeiten vor (vgl. z. B. Studien zur Phonetik des Thailändischen von Kelz/Kummer 1989), thaiistische linguistische Untersuchungen tendieren im Allgemeinen aber dazu, pragmatische Themen zu behandeln, wie z. B. Höflichkeit (Kummer 1990, 2006). Daneben gibt es einige relevante linguistische Untersuchungen, die zwar von deutschen Sprachwissenschaftlern durchgeführt wurden, die aber nicht den Sprachvergleich Deutsch-Thailändisch zum Gegenstand haben, sondern im Rahmen der Universalienforschung entstanden sind, so dass Thailändisch nicht mit Deutsch, sondern mit anderen, vor allem Nachbarsprachen, verglichen wird (vgl. z. B. Bisang 1992). Ebenfalls relativ häufig sind kontrastive Arbeiten erschienen, in denen das Deutsche mit zahlreichen anderen Sprachen verglichen wird. Vor allem das Mannheimer Institut für Deutsche Sprache hat in den letzten Jahren durch internationale Zusammenarbeit viele Vergleichsstudien durchgeführt, allerdings betreffen die behandelten Vergleichssprachen mehrheitlich europäische Sprachen (vgl. z. B. Zifonun 2001, 2007). Was die typologisch grundverschiedenen Sprachen betrifft, scheint das Japanische die mit dem Deutschen am häufigsten verglichene Sprache zu sein, wenn man z. B. das Projekt "Kontrastive Grammatik DeutschJapanisch" betrachtet, das bereits in den 1970er Jahren durchgeführt wurde (vgl. ausführlicher dazu Kanebo 2001).

Zur Beschreibung der Grammatik des Thailändischen, vor allem im Vergleich zum Englischen, liegt hingegen eine Reihe von Arbeiten vor, auf die zurückgegriffen werden kann (vgl. z. B. Noochoochai 1979, Sookgasem 1990). Es gibt zwar auch Vergleichsstudien mit anderen Sprachen wie z. B. Französisch, Japanisch, Chinesisch usw., deren Anzahl übersteigt aber kaum die Anzahl der thai-deutschen kontrastiven Studien.

\subsection{Typen der kontrastiven Studien und deren thematische Schwerpunkte}

Die hier behandelten Forschungsprojekte lassen sich nach ihrer Zielsetzung bzw. ihrer allgemeinen Ausrichtung in drei Gruppen kategorisieren:

- Kontrastive Analysen: Es handelt sich hierbei um Vergleiche unterschiedlicher Aspekte der beiden Sprachen. Behandelt wurden bisher die Themen Wortstellung (Sriuranpong 1997a), Internationalismen (Sriuranpong 1997b), stilistisch-rhetorische Gestaltung von Werbeslogans (Palabhut 2001), Negation (Ralugmool 2002), Relativsätze (Voraurai 2002), Verbvalenz und Satzstruktur (Laisanitseereekul 2003), Nominalkomposita (Suriyajun 2003), Ausdruck der Höflichkeit (Loto 2006) und Merkmale des Passivs (Sriuranpong 2007),

- Übersetzungsforschung: Im Rahmen solcher Studien werden hauptsächlich thailändische Entsprechungen einiger deutscher sprachlicher Elemente bzw. deren Übertragung ins Thailändische untersucht. Bereits untersucht wurden Modal- bzw. Abtönungspartikeln (Namsoongnein 1987, Saengaramrauang 2002), es (Benjawattananan 2006) und Passivsätze (Saengaramruang 2008),

- Studien zum Erwerb oder zur Vermittlung des Deutschen bei thailändischen Deutschlernenden: Diese Gruppe umfasst die restlichen hier besprochenen Arbeiten. Es handelt sich z. T. um empirische Untersuchungen, bei denen der Vergleich des Deutschen mit dem Thailändischen als Grundlage für die Analyse der empirischen Daten dient. Aufsätze, deren Ziel nicht der Sprachvergleich selbst ist, sondern die nur kontrastive Ansätze widerspiegeln, die wiederum für den Unterricht Deutsch als Fremdsprache für thailändische Lernende nutzbar gemacht werden können, werden ebenfalls zu dieser Gruppe gezählt. 
Die folgende Abbildung gibt einen Überblick über die 20 klassifizierten Arbeiten.

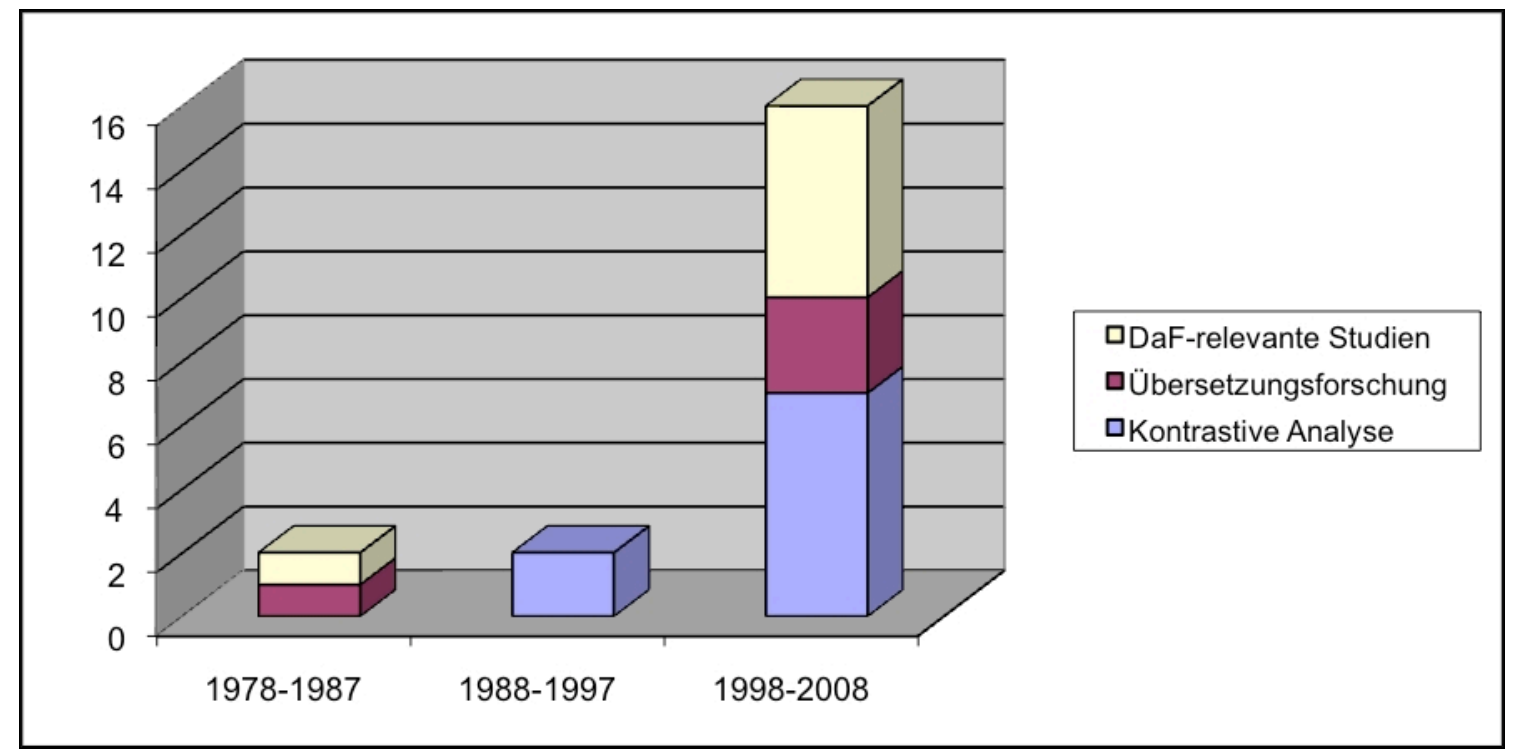

Anteile der Arbeiten des einzelnen Typs in drei unterschiedlichen Zeitstufen

Der erste Zeitraum (1978-1987) gilt als Pionierphase für Forschung in der Germanistik in Thailand. Dass die ersten kontrastiven Arbeiten Ende der 1970er Jahre entstanden, hängt damit zusammen, dass im Jahre 1974 der erste Masterstudiengang im Fach Deutsch eingeführt wurde. Anders als das Bachelorstudium, das in Thailand üblicherweise 4 Jahre dauert und stark auf die Verbesserung der sprachlichen Fertigkeiten der Studierenden abzielt, ist das Masterstudium wissenschaftlich orientiert, die thematischen Schwerpunkte sind allerdings mehrheitlich der deutschen Literaturwissenschaft zuzuordnen. Die einzige linguistische Masterarbeit im Bereich der Germanistik stammt von Narümon Ngaosuwan (1978) und thematisiert Hauptschwierigkeiten der thailändischen Studenten beim Erlernen der Zielsprache Deutsch. Der Fehleranalyse ihrer empirischen Daten liegt der Vergleich, vor allem die ausführliche Darstellung der phonologischen, morphologischen sowie syntaktischen Unterschiede zwischen der Ausgangs- und der Zielsprache, zugrunde. Obwohl der Titel der Arbeit selbst nicht direkt auf eine kontrastive Analyse hindeutet, bezeichnet die Autorin in ihrer Einleitung ihre Untersuchung ausdrücklich als "kontrastive Analyse". Die Arbeit ist als Pionierleistung im Bereich der kontrastiven Studien Deutsch-Thailändisch anzusehen und spiegelt die langjährige Erfahrung der Autorin im Bereich der Vermittlung der deutschen Sprache an thailändische Muttersprachler mit kontrastiven Ansätzen wider. Im Gegensatz zu dieser in Thailand verfassten Masterarbeit ist die kontrastive Untersuchung von Chutamat Namsoonnein (1987), die an der Universität Konstanz als Magisterarbeit angenommen wurde, der Kategorie "Übersetzungsforschung" zuzuordnen. In ihrer Studie werden Entsprechungen der deutschen Modalpartikeln denn, doch und mal untersucht. Bemerkenswert an dieser Arbeit ist es, dass sich die Verfasserin nicht nur auf ihre Sprachkompetenz als Muttersprachlerin des Thailändischen stützt, sondern auch erstmalig eine Korpusanalyse durchführt, bei der vier übersetzte literarische Werke berücksichtigt werden.

Die zweite Phase von 1988-1997 kann als eine Art Rückschritt im thailändischen Germanistikstudium betrachtet werden. Obwohl in diesem Zeitraum mehrere Arbeiten publiziert wurden und die Anzahl der thailändischen Germanisten, die in Deutschland promovierten (vgl. Watananguhn 2008: 41), eindeutig stieg, wurde in Thailand keine einzige Masterarbeit im Fach Deutsch geschrieben (s. ausführlicher Abschnitt 3.1). Allerdings wurden in dieser Zeit einige bedeutende Monographien verfasst, die bis heute unter thailändischen Deutschlernenden bekannt sind und häufig benutzt werden, so z. B. das Thai-Deutsche Wörterbuch 
von Otrakul et al. (1982) oder die am weitesten verbreitete Übungsgrammatik von Saengaramruang (1986-1991). Als einer der wichtigsten Schritte für den Deutschunterricht in Thailand gilt außerdem die Erarbeitung der ersten zwei Regionallehrwerke Viel Spaß mit Deutsch (Taatloha et al. 1989-1992) und Lesen macht Spaß (Watananguhn et al. 1988).

Die einzige kontrastive Untersuchung, die während dieses Zeitraums durchgeführt wurde, ist zugleich die wichtigste thai-deutsche linguistische kontrastive Studie. Die Dissertation von Sriuranpong (1997a), die die Wortstellung im Deutschen und im Thailändischen vergleicht, gilt immer noch als die ausführlichste kontrastive linguistische Untersuchung, in der das Sprachpaar Deutsch und Thailändisch behandelt wird. Sie ist außerdem die erste Arbeit, in der explizit auf die Forschungslücke in der thai-deutschen kontrastiven Linguistik hingewiesen wird. Diese Untersuchung gilt deshalb als Vorläufer und Vorbild für die meisten später durchgeführten linguistischen kontrastiven Analysen. Die Autorin selbst führte im Anschluss daran noch weitere kontrastive Untersuchungen durch (vgl. z. B. Sriuranpong 2007) und betreute einige in der nächsten Phase verfassten Masterarbeiten, die sich der kontrastiven Analyse Deutsch-Thailändisch widmen. Diese Arbeiten weisen z. T. ähnliche methodische Ansätze und Strukturen wie bei ihrem Vorläufer auf (vgl. Ralugmool 2002; Suriyajun 2003; Voraurai 2002).

Erst in der letzten Phase (1997-2008) haben kontrastive Studien Deutsch-Thailändisch einigermaßen Fuß gefasst, was u. a. sowohl an der Anzahl der Arbeiten an sich, an den unterschiedlichen Typen von Arbeiten als auch an den behandelten Aspekten und der angewendeten Methodologie ersichtlich wird. Auch wenn es insgesamt übertrieben wäre, von einer Blütezeit der kontrastiven germanistischen Forschung in Thailand zu sprechen, zeichnet sich doch deutlich eine positive Tendenz ab. Kontrastive Studien werden an den beiden Universitäten, an denen ein Masterstudiengang für Deutsch bzw. Deutsch als Fremdsprache angeboten wird, fortgesetzt, so dass immer mehr kontrastive Aspekte behandelt werden.

Zwei empirische Untersuchungen, die den Erwerb des Deutschen als Zweit- bzw. Fremdsprache behandeln, widmen sich auch einzelnen kontrastiven Aspekten der deutschen und der thailändischen Sprache. Im Mittelpunkt der Dissertation von Noraseth Kaewwipat (2007) steht die Anwendung einer sog. "Kontrastiven Lesegrammatik" im Unterricht Deutsch als Fremdsprache für thailändische fortgeschrittene Lernende. In der Untersuchung zum Hochdeutscherwerb durch thailändische Immigrantinnen in der Deutschschweiz (Attaviriyanupap 2007) dient die kontrastive Studie Deutsch-Thailändisch hingegen nicht der Didaktisierung, sondern der linguistischen Analyse der Lernersprache. Auch die in Attaviriyanupap (2007) gewonnenen Erkenntnisse können im Deutschunterricht für thailändische Muttersprachler nutzbar gemacht werden. Die Habilitationsschrift von Salifou Traoré (2008) widmet sich im Gegensatz zu diesen beiden Dissertationen nicht der Analyse empirisch gewonnener Daten, sondern befasst sich konzeptionell mit zwei ausgesuchten grammatischen Phänomenen (Tempus und Genus Verbi) und zeigt, inwiefern der Vergleich Deutsch-Thailändisch in Form einer "Interkulturellen Grammatik" für den Unterricht Deutsch als Fremdsprache von Bedeutung ist und wie sie dafür eingesetzt werden könnte.

In den meisten kontrastiven Untersuchungen Deutsch-Thailändisch stehen die verschiedenen Kernbereiche eines Sprachsystems im Mittelpunkt, vor allem die syntaktischen Aspekte wie etwa Wortstellung, Verbvalenz und Satzstruktur, Relativsätze, Negation, Genus Verbi sowie Tempus. Im Bereich der Morphologie ist mit einer Untersuchung zu Nominalkomposita (Suriyajun 2003) bereits eine erste Arbeit entstanden. Da das Thailändische als isolierende Sprache nicht sehr formenreich ist, bieten sich kontrastive Studien nicht unbedingt an; die eigentlichen Forschungslücken bestehen vor allem in den Bereichen der Phonetik/Phonologie und Morphosyntax/Syntax. Obwohl bisher in den Bereichen Phonetik/Phonologie, Morphologie, Lexik und Syntax einzelne Teilaspekte als Untersuchungsgegenstände der kontrastiven 
Studien Deutsch-Thailändisch behandelt wurden, kann man auf dem gegenwärtigen Stand den Schluss ziehen, dass nur die Modalpartikeln (Ngamsoonein 1987; Saengaramruang 2002), Wortstellung (Sriuranpong 1997) sowie das Passiv (Sriuranpong 2007, Saengaramruang 2008, Traoré 2008) ziemlich gut untersucht werden. Die zentralen verbalen Kategorien wie Tempus und Modus, die im Thailändischen fehlen und beim Vergleich mit dem Deutschen mit den Oberbegriffen "Temporalität" und "Modalität" bezeichnet werden müssen, werden kaum behandelt (vgl. Traoré 2008) oder bleiben vorwiegend auf einmorphemische Wörter beschränkt (vgl. z. B. Modalpartikeln bei Namsoongnein 1987, Saengaramruang 2002 oder "es" bei Benjawattananan 2006). Der Behandlung komplexer Elemente wie flektierter oder deklinierter Wortformen wird hingegen kaum Beachtung geschenkt. Weitere Studien sollten sich m. E. deshalb möglichst mit unterschiedlichsten Aspekten im nominalen wie auch im verbalen Bereich befassen, weil sie zentral für die Flexion im deutschen System sind und sowohl formalen als auch konzeptionellen Unterschieden zwischen dem Deutschen und dem Thailändischen zugrunde liegen.

Es ist anzumerken, dass die meisten Studien praxisorientiert sind. D. h., ihr Hauptziel ist es, die kontrastive Analyse entweder für den Deutschunterricht für thailändische Lernende oder für die Übersetzungsarbeit von Deutsch ins Thailändische nutzbar zu machen. Es fehlt noch an linguistischer Grundlagenforschung, die die beiden Sprachen an sich ausführlich behandeln, ohne Ergebnisse, die im DaF-Unterricht für thailändische Lernende direkt anwendbar sind.

\subsection{Angewandte Methodologie}

Jede der drei erwähnten Kategorien der kontrastiven Studien weist eine bestimmte Methodologie auf. Die erste Gruppe, die direkte kontrastive Analyse, ist methodisch systemlinguistisch und deskriptiv orientiert. Zum Teil beruhen auch pragmatische und stilistische Aspekte wie Höflichkeit (Loto 2006) oder Rhetorik (Palabhut 2001) auf der Analyse von grammatischen Strukturen. Meistens werden Form, Funktion und Bedeutung bestimmter sprachlicher Phänomene bzw. Elemente theoretisch behandelt und anhand von Beispielsätzen beschrieben. Anzumerken ist, dass die Quellenangabe der Beispielsätze in den ersten Untersuchungen meistens fehlt, während jene in den späteren Studien mehrheitlich belegt sind. Außerdem lässt sich feststellen, dass jüngere Untersuchungen häufiger korpusbasiert sind und die beiden Sprachen systematischer untersucht werden, was eine positive Entwicklung im Hinblick auf die Methodologie darstellt. Vor allem die Tatsache, dass die kontrastiven Analysen großteils auf empirischen Daten und nicht mehr auf der Intuition der Verfasser als Muttersprachler einer Sprache basiert, erhöht die Objektivität der Untersuchungen. Da es relativ schwierig ist, für kontrastive Studie geeignete vergleichbare Daten bzw. Korpora zu finden, vor allem wenn Sprecher der jeweiligen Sprachen räumlich und kulturell sehr weit voneinander entfernt sind, beschränken sich die verwendeten Korpora deshalb noch hauptsächlich auf gängige Grammatiken (vgl. Laisanitsereekul 2003) sowie auf ausgewählte Konstruktionen in größeren Korpora (vgl. Sriuranpong 2007), so dass noch keine umfassende, korpusbasierte quantitative Analyse vorliegt.

Im Bereich der Übersetzungsforschung hingegen gehörten Korpusanalysen von Beginn an zum Methodeninventar (vgl. z. B. Namsoongnein 1987), z. T. kamen Korpusanalysen auch bei quantitativen Untersuchungen zum Einsatz (vgl. z. B. Benjawattananan 2006). Dadurch können Entsprechungen bestimmter sprachlicher Elemente systematisch und präzise untersucht werden. Im Bereich der Übersetzungsforschung mangelt es auch nicht an geeigneten Daten (d.h. an bilingualen Korpora), allerdings wurde bisher jeweils nur die Übersetzung in eine Richtung untersucht, nämlich die Übersetzung vom Deutschen ins Thailändische. Die Untersuchung der Gegenrichtung wäre als eine wichtige zusätzliche Dimension der kontrastiven Analyse wünschenswert. 
Schließlich beruhen auch die meisten DaF-relevanten Untersuchungen auf empirischer Methodologie. In diesem Bereich werden meistens Korpora der Lernersprache zusammengestellt und diese empirischen Daten werden je nach Zielsetzung der jeweiligen Studie ausgewertet und analysiert. Die Datengewinnung der bisher durchgeführten Studien erfolgte jedoch jeweils sehr unterschiedlich, z. B. durch Sammlung von Aufsätzen (Ngaosuwan 1978) oder durch Aufnahme authentischer Gesprächssituationen (Attaviriyanupap 2007). Die Beispielsätze im kontrastiven Teil dieser Arbeiten werden jedoch nicht belegt, was damit zusammenhängt, dass die thai-deutschen linguistischen kontrastiven Analysen zwar jeweils integraler Bestandteil dieser Arbeiten sind, dass der Sprachvergleich jedoch nicht das Hauptziel der Untersuchungen ist, wodurch sich die Zusammenstellung eines zusätzlichen Korpus erübrigt.

Korpusbasierte Studien bringen in der Tat viele Vorteile mit sich. Die Anwendung guter Korpora macht die Untersuchung von Sprachen, die aus linguistischer Sicht immer als ein "System" zu betrachten sind, besser fassbar und ermöglicht auch quantitative Analysen, die in empirischen Studien häufig von Bedeutung sind, weil quantitative Ergebnisse falsifizierbar sind und nicht ausschließlich auf der subjektiven Interpretation der Forscher beruhen. Dies ist einer der Gründe dafür, warum im Bereich der kontrastiven Linguistik sowie in der Übersetzungsforschung vermehrt korpuslinguistische Methoden eingesetzt werden (Granger et al 2003). Vor allem das von Johansson (2003: 39) beschriebene Modell eines "parallel corpus", nach dem jeweils Original- und Übersetzungstexte der zu vergleichenden Sprachen miteinbezogen werden, scheint m. E. ein guter Ansatz für weitere kontrastive Untersuchungen zu sein.

\section{Faktoren, die zum Gesamtbild der Forschungslage in der thai-deutschen kontrastiven Linguistik beitragen}

\subsection{Erweiterung des thailändischen Germanistikstudiums durch Master- und Promotionsstudiengänge}

Dass die Germanistik in Thailand ihre Wurzeln in der deutschen Germanistik hat, steht außer Frage, zumal das erste Bachelor-Curriculum (BA) hauptsächlich von deutschen Lektoren entworfen wurde (vgl. Watananguhn 2008: 36). Allerdings unterscheidet sich das BA-Studium sowohl formal als auch inhaltlich stark von einem Germanistikstudium im deutschsprachigen Raum: Während das Studium der Germanistik im deutschsprachigen Raum ursprünglich in der Regel ein Studium der deutschen Linguistik und Literatur mit einem Magisterabschluss war, war das thailändische Germanistikstudium von Beginn an am amerikanischen System ausgerichtet und somit in zwei Stufen geteilt, wobei in der Anfangsphase nur die BA-Stufe angeboten wurde. Auch nach der Bologna-Reform im deutschsprachigen Raum, die die Einführung von Bachelor- und Masterstudiengängen mit sich brachte, lassen sich - schon allein aufgrund der Tatsache, dass es sich beim Germanistikstudium in Thailand um ein Studium für Auslandsgermanisten handelt - große Unterschiede erkennen. Im Vergleich zu Studienanfängern der Germanistik im deutschsprachigen Raum, die meist Muttersprachler des Deutschen sind, sind für Germanistikstudenten in Thailand, vor allem in den ersten zwei Studienjahren, fast lediglich fertigkeitsorientierte Sprachkurse vorgesehen. Dass in Thailand heutzutage auch Studiengänge über der BA-Stufe angeboten werden, trägt deshalb erheblich zur besseren wissenschaftlichen Lage der Germanistik bei. ${ }^{4}$ Erst auf dieser Stufe können

\footnotetext{
4 Insgesamt werden drei Studienprogramme auf einem Graduiertenniveau, d. h. für Absolventen von BachelorStudiengängen, eingeführt. An der Chulalongkorn Universität gibt es seit 1974 ein Masterprogramm (MA) für Deutsch und seit 2006 zusätzlich einen Promotionsstudiengang (Ph.D). An der Ramkhamhaeng Universität wird seit 1999 ein Studienprogramm mit MA-Abschluss in Deutsch als Fremdsprache angeboten.
} 
Interessenten für deutsche Linguistik und Literatur ihr Wissen vertiefen und auf diesem Gebiet etwas Konkretes erforschen. Aufgrund der Tatsache, dass es in Thailand erst seit relativ kurzer Zeit mehrere germanistische MA-Studiengänge gibt, ist es auch nicht verwunderlich, dass es sich bei den im vorigen Abschnitt besprochenen kontrastiven Studien mehrheitlich um Abschlussarbeiten handelt, die im Rahmen eines Masterstudiums in Thailand angefertigt wurden.

Die wissenschaftlichen Ansprüche an Magisterarbeiten sind im Allgemeinen nicht so hoch, wie dies bei Dissertationen oder einzelnen selbstständigen Forschungsprojekten der Fall ist. Eine Masterarbeit zu schreiben dient in der Tat vielmehr als Übung und Grundlage für weitere Forschung. Das Niveau der Masterarbeiten, die an thailändischen Universitäten entstehen, wird darüber hinaus bedauerlicherweise durch eine Regelung im thailändischen Hochschulsystem beeinträchtigt, die vor fast 20 Jahren eingeführt wurde. Gemäß dieser Regelung haben Masterstudenten mehrerer Fachbereiche, einschließlich der Germanistik, die Möglichkeit im Rahmen ein und desselben Studienprogramms zwischen "Plan A" mit einer "Thesis" und "Plan B" mit einem "Special Project" wählen dürfen. Während die "Thesis" bzw. Masterarbeit mit einer "Magisterarbeit" im deutschsprachigen Raum einigermaßen vergleichbar ist, wird eine schriftliche Arbeit im Rahmen von einem "Special Project" als eine "kleinere Arbeit" bezeichnet, deren Umfang und Aufwand etwa einer Bachelorarbeit im deutschsprachigen Raum entspricht. ${ }^{5}$ Meines Erachtens hat die Einführung dieser "kleinen" Arbeiten zu einem Rückschritt im Forschungsbetrieb der thailändischen Germanistik geführt. Bei der Bestandsaufnahme aller thai-deutschen kontrastiven linguistischen Studien, wobei alle in Thailand im Fach Deutsch angefertigten Masterarbeiten mitberücksichtigt wurden, zeigt sich, dass lange Zeit, nämlich zwischen 1985 und 1999, keine einzige "Thesis" verfasst wurde. Dies bedeutet allerdings keinesfalls, dass es in diesem Zeitraum keine Germanistik-Absolventen gab oder keine schriftlichen Arbeiten angefertigt wurden, sondern dass diese Arbeiten nicht dokumentiert wurden. Während alle Arbeiten, die in Thailand als "Thesis" angenommen werden, veröffentlicht werden müssen, indem die Verfasser bei der thailändischen Nationalbibliothek die ISBN-Nummer beantragen und zumindest ein Exemplar der Arbeit der Zentralbibliothek der jeweiligen Universität zur Verfügung stellen müssen, fehlt diese Verpflichtung bei den im Rahmen eines "Special Project" verfassten Arbeiten. Diese Neuregelung hatte zwei Konsequenzen: Erstens wurde der Wissenschaftsbetrieb der thailändischen Germanistik dadurch nicht genügend gefördert. Den Masterstudenten wie auch den Wissenschaftlern, die weitere Studien durchführen wollten, fehlten Ergebnisse aus den früheren relevanten Studien. D. h., eine gründliche Recherche wäre nur durch persönliche Kontakte möglich, weil die Titel sowie die Abstracts dieser Arbeiten nirgendwo systematisch dokumentiert werden. Nicht selten können sich die Personen, die in diesem Zeitraum selbst eine solche "kleine" Arbeit geschrieben haben, nicht mehr an den Titel ihrer Arbeit erinnern. Es fehlt hier also die "Schriftkultur", die eigentlich ein wichtiger Bestandteil zur Förderung der Wissenschaft in allen Fachbereichen ist. Das hier zusammengefasste Gesamtbild der Forschungslage in der thailändischen Germanistik in der zweiten Phase (s. 2.1 und 2.2) spiegelt deshalb nicht die eigentliche Leistung der thailändischen Germanisten wider. Zweitens führt die Verkleinerung der Masterarbeit im traditionellen Sinne m. E. dazu, dass einige Arbeiten die behandelten Forschungsfragen weder umfassend noch tiefgreifend beantworten können. Die Verfasser dieser Arbeiten scheinen sich aufgrund der Rahmenbedingungen auf einen "kleinen" Umfang beschränkt zu haben, obwohl ihre Forschungsthemen bzw. die Forschungsgegenstände an sich viel zu groß und kompliziert sind, als dass man sie im Rahmen einer kleinen Arbeit genügend

\footnotetext{
5 Der Unterschied liegt in der Zahl der Kreditpunkte und bei einigen Fächern, wie auch im Fach Deutsch, in der minimalen Seitenzahl der Arbeiten, die man schreiben muss. Deshalb wird sie unter Germanisten in Thailand auch ab und zu als "Kleine Magisterarbeit" bezeichnet.
} 
behandeln könnte. Größere Projekte, die solche kleinen Arbeiten wiederum synthetisieren, wären deswegen sinnvoll.

Bemerkenswert ist auch die Tatsache, dass alle Masterarbeiten im Fach Deutsch oder Deutsch als Fremdsprache nur in deutscher Sprache verfasst werden dürfen, obwohl einige Arbeiten inhaltlich nur den Lesern dienen, die die thailändische Sprache beherrschen. Es handelt sich dabei vor allem um Arbeiten, die der Übersetzungsforschung zugeordnet werden. Es wäre notwendig, dass alle Masterarbeiten, die in Thailand entstehen, je nach inhaltlicher Ausrichtung auf Deutsch, auf Thailändisch oder auch auf Englisch verfasst werden dürfen. Es gibt nur wenige Arbeiten, deren Ergebnisse in thailändischer Sprache publiziert werden, wie z. B. die Übersetzungsstudien zu Abtönungspartikeln und Passivsätzen (Saengaramruang 2002, 2008), und die dadurch ebenfalls für thailändische Übersetzer ohne Deutschkenntnisse zugänglich und nützlich sind. Würde Deutsch nicht als obligatorische Sprache der Masterarbeit festgelegt, könnte ein Austausch zwischen thailändischen Germanisten und anderen thailändischen Sprachwissenschaftlern und Übersetzern ermöglicht werden. Dass Germanistikmasterstudenten verpflichtet sind, ihre Arbeit in deutscher Sprache zu verfassen, auch wenn sich die Arbeit vor allem dem Thailändischen widmet und für diejenigen, die Thailändisch nicht lesen können, unverständlich ist, kann m. E. auf die konzeptionelle Ausrichtung der Germanistik in Thailand zurückgeführt werden. Das Germanistikstudium in Thailand scheint sich auf die Vermittlung der deutschen Sprache und Literatur zu beschränken, und schenkt der fertigkeitsorientierten Sprachkompetenz der Lernenden große Beachtung. Saengaramraung (2007: 355f.) beschreibt beispielsweise den Charakter des thailändischen Germanistikstudiums wie folgt:

Die Auslandsgermanistik hat andere Schwerpunkte gesetzt als die traditionelle Germanistik, insofern sie auf Lerner des Deutschen fokussiert ist. Das gilt natürlich auch für die Germanistik in Thailand:

1. Deutsch als Fremdsprache (Methode und Didaktik der Sprachlehre für Ausländer)

2. Forschungen über die deutsche Sprache und Erteilung von Lehr- und Handbüchern im Hinblick auf die Vermittlung der deutschen Sprache an eine bestimmte Lernergruppe.

Der wissenschaftliche Betrieb der thailändischen Germanisten sollte deshalb neu definiert werden oder anders gesagt zurück zu ihren Wurzeln gehen. Die Germanistik in Thailand richtete sich anfangs auch weitgehend nach der deutschen Germanistik (Wattananguhn 2008: 36), bevor das Fach "Deutsch als Fremdsprache" überhaupt aufkam und in den letzten dreißig Jahren seine Blütezeit genießt. Der Begriff "Auslandsgermanistik" wird heute anscheinend zu häufig mit "Deutsch als Fremdsprache" gleichgesetzt. Ursprünglich wurde er mit Bezug auf die "Inlandsgermanistik" definiert und hatte eine andere Bedeutung: Man verstand darunter auch in Thailand - wissenschaftliche Forschung bzw. Beiträge von Nicht-Muttersprachlern des Deutschen, die sich im Ausland mit der deutschen Sprach- oder Literaturwissenschaft eingehend befassen. Unter dem Dach der "Auslandsgermanistik" muss viel Verschiedenes Platz finden wie z. B. Sprachdidaktik, Landeskunde, Literaturwissenschaft und Linguistik. ${ }^{6}$ Die Auslandsgermanistik kann daher viel zur Inlandsgermanistik beitragen, indem sie durch das Anderssein der Wissenschaftler fremde Perspektive einbringt, die bei Muttersprachlern fehlen können oder von ihnen noch nie wahrgenommen wurden, wodurch sich der Forschungsgegenstand der Germanistik umfassender behandeln lässt. Insofern beschränkt sich die Forschung auf diesem Gebiet nicht nur auf den Deutschunterricht für NichtMuttersprachler. Die Inlandsgermanistik kann von diesem "fremden Blick" nur profitieren, denn nicht selten ist der Blick von Außen schärfer und bewusster (vgl. dazu auch Krumm 2003: 259f.)

\footnotetext{
6 Der Frage nach der "Auslandsgermanistik" wird beispielsweise bei Lepper (2008) nachgegangen.
} 


\subsection{Wissenschaftliche Zeitschriften und Tagungen}

Ein bedeutender Faktor zur Erhöhung der Anzahl der in Thailand durchgeführten Forschungsprojekte im Bereich der Germanistik und Deutsch als Fremdsprache sind einerseits die Herausgabe von zwei Fachzeitschriften und andererseits die regelmäßige Durchführung von thailändischen Germanistentreffen. Neben den anderen thailändischen Fachzeitschriften, die von allen Philosophischen Fakultäten thailändischer Universitäten publiziert werden, gibt es seit über 10 Jahren zwei wichtige Zeitschriften, die sich den Publikationen thailändischer Masterstudenten, Deutschlehrern und Germanisten widmen:

- Die Zeitschrift des Thailändischen Deutschlehrerverbands: Diese Zeitschrift wurde zum ersten Mal im Jahre 1996 unter dem Titel "Siam-Forum" veröffentlicht. Im Jahr danach wurde sie in "TDLV-Forum. Zeitschrift für Sprache, Literatur und Kultur" umbenannt. Sie wird seitdem einmal jährlich herausgegeben. In dieser Zeitschrift werden sowohl deutsch- als auch thaisprachige Aufsätze über die deutsche Sprache, Literatur und Kultur publiziert.

- "Die Brücke. Zeitschrift für Germanistik in Südostasien": Parallel zum Masterstudiengang in Deutsch als Fremdsprache wird diese Zeitschrift seit 2002 von der Deutschabteilung der Ramkhamhaeng Universität herausgegeben. Jährlich werden 1 bis 2 Hefte veröffentlicht. Alle Beiträge werden in deutscher Sprache verfasst. Auch Beiträge der germanistischen Wissenschaftler aus den Nachbarländern in Südostasien, vor allem aus Vietnam, werden hier regelmäßig publiziert.

Im Allgemeinen lässt sich kein inhaltlicher Unterschied zwischen den beiden Fachzeitschriften feststellen. Die Beiträge behandeln relevante Themen im Bereich des Deutschunterrichts sowie der deutschen Linguistik und Literatur. Ein kleiner Unterschied besteht jedoch darin, dass Die Brücke formal stärker einer internationalen Fachzeitschrift ähnelt, weil sie in jedem Heft weniger Beiträge beinhaltet, die hauptsächlich von wissenschaftlichen Studienergebnissen berichten, während das TDLV-Forum z. T. Berichte über Aktivitäten der thailändischen Deutschlehrer umfasst.

Ein anderer Faktor, der die Germanistik in Thailand fördert, ist die Veranstaltung von germanistischen Tagungen in regelmäßigen Abständen. Bis zum Ende der 1990er Jahre fand in Thailand jährlich zu verschiedenen Themen ein wissenschaftliches Treffen in Form eines sog. "Nachkontaktseminars" statt, das durch Unterstützung des DAADs (Deutscher Akademischer Austauschdienst) und $\mathrm{ab}$ und $\mathrm{zu}$ in Zusammenarbeit mit dem Goethe-Institut ermöglicht werden konnte. Im Jahr 1999 wurde dann das bis jetzt größte Germanistentreffen in Bangkok organisiert, an dem Germanisten aus Deutschland, Indien, Indonesien, den Philippinen, Taiwan, Thailand und Vietnam teilnahmen. Dieses Treffen gilt als Auftakt für die später unter dem Titel "Thailändisches Germanistentreffen" veranstalteten Tagungen, da dieses Treffen zu einem fruchtbaren interkulturellen Austausch führte. ${ }^{7}$

Das thailändische Germanistentreffen, deren Teilnehmer sich mehrheitlich aus Germanisten in Thailand zusammensetzen, findet seit 2001 in einem regelmäßigen Turnus von zwei Jahren statt. Bis jetzt wurden bereits 4 Treffen veranstaltet, wobei jeweils eine Universität mit Deutsch-Studienprogramm(en) abwechselnd als Gastgeber fungierte. Die Möglichkeit zur Präsentation eigener Forschungsergebnisse motiviert Deutschlehrer und Germanisten in

7 Beispielsweise wurde einer der Teilnehmer dieser Tagung, Prof. Vridhagiri Ganeshan aus Hyderabad, im Anschluss an das Treffen von einem durch den Thailand Research Fund unterstützten Projekt als Gastreferent zweimal nach Thailand eingeladen. Außerdem hat er einen Beitrag bei der thailändischen Zeitschrift $T D L V$ Forum publiziert (vgl. Ganeshan 2000). 
Thailand gleichermaßen, mehr eigenständige Forschungsarbeit zu leisten, über die sie sich mit ihren Fachkollegen austauschen können. Obwohl eine solche wissenschaftliche Veranstaltung bisher nur mit der Unterstützung von deutscher Seite möglich ist, gilt sie als ein gutes Zeichen für die Weiterentwicklung der Germanistik in Thailand. Sie fördert eindeutig die wissenschaftliche Vernetzung und trägt zur allgemeinen Verbesserung des Deutschunterrichts und der Germanistik in Thailand bei.

\subsection{Forschungsarbeit der thailändischen Germanisten}

Obwohl in der letzten Zeit, vor allem in den letzten 10 Jahren, viele Forschungsarbeiten entstanden sind, lässt sich die Tendenz feststellen, dass sich nur sehr wenige thailändische Germanisten mit Forschungsprojekten beschäftigen. Die vorliegende Bestandsaufnahme zeigt bei den besprochenen kontrastiven Arbeiten, abgesehen von den Verfassern von Masterarbeiten, nur wenige Namen von Forschern. Dieser Befund ist symptomatisch für die Situation der Germanistik in Thailand. Es stellt sich deshalb die Frage, worauf diese Situation zurückzuführen ist. Einer der Gründe dafür, dass sich nur sehr wenige Germanisten der Forschung widmen, ist mit Sicherheit, dass die meisten Germanisten in Thailand nicht nur Wissenschaftler, sondern vor allem auch Sprachlehrer sind. Vor allem an den Universitäten, an denen das Germanistikstudium nur auf der BA-Stufe angeboten wird, werden fast nur Kurse durchgeführt, die auf die Verbesserung der sprachlichen Fertigkeiten der Lernenden abzielen. Die Dozierenden haben ein sehr hohes Lehrdeputat und müssen zahlreiche Hausaufgaben und Übungen korrigieren, so dass ihnen nur wenig Zeit für Forschungsarbeit bleibt. Darüber hinaus zeichnet sich in letzter Zeit die Tendenz ab - und dies gilt für den Deutschunterricht in Thailand im Allgemeinen -, dass es immer weniger fortgeschrittene Deutschlernende gibt, während die Zahl der Anfänger eindeutig steigt. Dies betrifft sowohl die Zahl der Deutschlernenden an Bildungsinstitutionen und Universitäten ${ }^{8}$ als auch die Teilnehmerzahl der außerschulisch und außeruniversitär angebotenen Kurse wie etwa am Goethe-Institut. Da der Bedarf dort groß ${ }^{9}$ ist, müssen viele Deutschdozenten mithelfen, Deutschkurse für Anfänger, und teilweise auch für lernungewohnte Personen, zu leiten. Es steht aus diesem Grund außer Frage, dass viele von ihnen weder Zeit noch Kraft haben, um sich für Forschungsarbeit einzusetzen. Vor diesem Hintergrund lässt sich schlussfolgern, dass die Lage der Publikationen wie auch die durchgeführten Forschungsprojekte nicht das eigentliche wissenschaftliche Leistungspotential der thailändischen Deutschlehrer und Germanisten widerspiegelt.

Viele in Thailand tätige Deutschdozenten, die als Auslandsgermanisten fungieren sollen, geraten in die Situation, dass sie sich ausschließlich fertigkeits- und berufsorientierter Lehre widmen müssen, da diese für ihre Absolventen wichtiger zu sein scheint als linguistische oder literaturwissenschaftliche Kenntnisse. Das große Engagement im Bereich des Sprachunterrichts ist oft nur auf Kosten der wissenschaftlichen Tätigkeit möglich. Trotzdem sollten germanistische Dozenten ihre Forschungsarbeit nicht völlig vernachlässigen. Die Forschung gilt nach wie vor als eine der Hauptmissionen aller Universitätsdozenten weltweit. Diejenigen thailändischen Germanisten, die regelmäßig Forschungsprojekte durchführen, sollen daher als gute Vorbilder für die anderen dienen und diejenigen, die noch über wenig Forschungs-

\footnotetext{
8 Momentan ist die Tendenz zu beobachten, dass immer mehr Lernende an asiatischen Sprachen - vor allem an Chinesisch -, interessiert sind, die in den letzten Jahren auch in Schulen gelehrt werden. Dies führt dazu, dass immer weniger Studierende mit Deutschkenntnissen ein Germanistikstudium aufnehmen. Viele der Deutschstudenten beginnen deshalb erst an der Universität mit dem Deutscherwerb (vgl. Akkramas 2008).

9 Eine Ursache dafür, warum die Zahl der Teilnehmer an Anfängerkursen des Goethe-Instituts enorm gestiegen ist, betrifft die obligatorischen Deutschkenntnisse der Visumsantragsteller, die durch ihre Mischehe mit deutschen Staatsangehörigen nach Deutschland emigrieren wollen.
} 
kompetenz bzw. -erfahrung verfügen, zur Forschung anlocken, z. B. durch gemeinsame Forschungsprojekte.

\subsection{Wissenschaftliche Zusammenarbeit}

Die wissenschaftliche Zusammenarbeit zwischen thailändischen Auslands- und Inlandsgermanisten ist zwar intensiv, sie ist aber immer hierarchisch organisiert ist, und zwar nur in der Konstellation "(Deutsche als) Geber und (Thailänder als) Nehmer"; die beiden Seiten sind nie gleichberechtigte Austauschpartner. Von Anfang an spielt die deutsche Partie (vertreten durch wichtige Organisationen wie z. B. den DAAD und das Goethe-Institut) eine dominierende Rolle, weil sie Stipendien vergibt, und deutsche Experten nach Thailand entsandte, um die hiesigen Deutschdozenten und -lehrer aus- und fortzubilden. Umgekehrt machen thailändische Studenten und Wissenschaftler öfter Aufenthalte im deutschsprachigen Raum, um sich aus- und fortbilden zu lassen. Es handelt sich also überwiegend um einen EinwegWissenstransfer. Eine tatsächliche Zusammenarbeit wie z. B. im Rahmen von gemeinsamen Forschungsprojekten, an dem thailändische und deutsche Kollegen auf gleichberechtigter Basis zusammenarbeiten, lässt sich kaum verwirklichen. Anzumerken ist außerdem, dass ein wichtiges Organ zur Förderung von internationaler wissenschaftlicher Zusammenarbeit wie die Alexander-von-Humboldt-Stiftung, die in Thailand selbst ein gutes und aktives Netzwerk von Wissenschaftlern verschiedener Fachgebiete, vor allem im Bereich der Naturwissenschaften, zustande gebracht hat, in der thailändischen Germanistik überhaupt keine Rolle spielt.

Die in Thailand vertretene deutsch-thailändische kontrastive Forschung wie auch die Forschungslage der thailändischen Germanistik im Allgemeinen sind eindeutig nicht durch groß angelegte Projekte geprägt, an denen Mitarbeiter aus verschiedenen Institutionen oder aus verschiedenen Fachgebieten teilnehmen, sondern vielmehr durch einzelne Forschungsvorhaben (häufig im Rahmen von Studienprogrammen) und vor allem durch eine sehr geringe Anzahl von Wissenschaftlern gekennzeichnet. Was in der thailändischen Germanistik eindeutig fehlt, ist deshalb die wissenschaftliche Zusammenarbeit auf allen Ebenen: zwischen verschiedenen Hochschulen und Universitäten, zwischen Germanisten und Linguisten wie auch Literaturwissenschaftlern anderer Sprachen und schließlich zwischen den thailändischen und anderen Auslands- sowie Inlandsgermanisten.

\section{$4 \quad$ Ausblick}

Um zwei Sprachsysteme linguistisch zu vergleichen, müssen möglichst viele Aspekte, oder im Idealfall das ganze System, berücksichtigt werden. In Anbetracht der Anzahl der bisher durchgeführten kontrastiven Studien Deutsch-Thailändisch, ist nicht zu übersehen, dass hier noch ein Desiderat besteht. Deshalb müssen als erstes sowohl die Anzahl als auch die Qualität der kontrastiven Studien gesteigert werden. Die kontrastive Linguistik Englisch-Deutsch könnte beispielsweise eine Vorbildfunktion übernehmen. Bereits vor über 20 Jahren lag für diese beiden Sprachen eine sehr große Anzahl an kontrastiven Studien vor (vgl. Keul 1981) und seitdem sind kontinuierlich zu verschiedenen Aspekten kontrastive Studien durchgeführt worden (vgl. hierzu ausführlicher König 2001). Die dadurch gewonnenen Erkenntnisse dienen als gute Grundlagen, von denen nutzbare Inhalte für die Erstellung von Grammatiken, Lehrwerken, Wörterbüchern abgeleitet werden konnten.

Die Geschichte der kontrastiven Linguistik in Japan zeigt, dass sich die kontrastive Grammatik in den 1970er Jahren auf einem pseudo-linguistischen Irrweg befand, weil sie nicht in der Lage war, erstens zur internationalen Universalienforschung substantiell beizutragen, zweitens ihre Beschreibungen erfolgreich in die Didaktik umzusetzen und drittens sich zu einem Teilbereich der Sprachtypologie zu entwickeln (Kaneko 2001: 451). In der Tat kann 
eine kontrastive Analyse zweier Sprachen nicht nur zur Verbesserung des Unterrichts Deutsch als Fremdsprache, sondern auch zur Beschreibung einer Einzelsprache beitragen. Der "Dialog der Grammatiken" hilft oft dabei, das Besondere einzelner grammatischer Phänomene zu erfassen und die grammatische Beschreibung in einen größeren Zusammenhang einzubetten. Die daraus gewonnenen Erkenntnisse können darüber hinaus für die Universalien- sowie Typologieforschung nutzbar gemacht werden. Jedoch braucht man bei so einer ehrgeizigen Zielsetzung einen lebhafteren Dialog zwischen allen relevanten Fachbereichen. Erwünscht wäre v. a. vermehrter Austausch zwischen den folgenden drei Gruppen: Praktikern auf dem Gebiet Deutsch als Fremdsprache mit unterschiedlichen Muttersprachen der Lernenden, Linguisten mit solidem Wissen in einer bestimmten Sprache (z. B. Deutsch und Thailändisch) sowie mit Typologie- und Universalienforschern.

Während sich in Thailand die Komparatistik bzw. die "Vergleichende Literaturwissenschaft" gut etabliert hat, ist die Lage der "Kontrastiven Linguistik" noch weit vom Status eines selbstständigen Faches entfernt. Ein Grund dafür könnte sein, dass sich die deutschthailändischen linguistischen kontrastiven Studien bisher zu sehr an der Methodologie des Deutschen als Fremdsprache orientieren. D. h., im Mittelpunkt steht immer der direkte Nutzen für den DaF-Unterricht für thailändische Lernende, was m. E. eine zu enge Zielsetzung ist. Das Ziel der kontrastiven Linguistik braucht nicht ausschließlich in der Planung von Fremdsprachenunterricht gesehen zu werden, sondern kann beispielsweise auch zur Typologie beitragen. Es ist sicherlich nicht zu leugnen, dass die Vermittlung der deutschen Sprache an thailändische Lernende von großer Bedeutung ist. Allerdings fehlt in der thailändischen kontrastiven Forschung die Suche nach linguistischen Erkenntnissen an sich bzw. Grundlagenforschung, die zur Germanistik im Allgemeinen wie auch zur Typologie- oder Universalienforschung beitragen und umgekehrt von ihnen profitieren können. Denn erst in einem solchem Kontext könnte die thailändische Germanistik international mit der Inlandsgermanistik wie auch mit der Auslandsgermanistik in allen anderen Ländern weltweit in einen echten wissenschaftlichen Diskurs treten. Erst dann können sich die kontrastive Linguistik (hier mit dem Sprachpaar Deutsch-Thailändisch), die Typologie- und Universalienforschung, die Beschreibung eines einzelnen Sprachsystems (hier Deutsch und Thailändisch) unterstützen und wechselseitig erhellen. Solche übereinzelsprachlichen Erkenntnisse können nicht nur für den Deutschunterricht und für thailändische Muttersprachler nutzbar gemacht werden, sondern können auch für den Thaiunterricht für deutsche Muttersprachler wie auch für die deutsche und die thailändischen Sprachwissenschaft im Allgemeinen von großem Nutzen sein.

\section{Literatur}

Akkramas, Pakini (2008): "Deutsch erst an der Uni? Ein Diskussionsbeitrag zur allgemeinen Tendenz des Deutschlernens in Thailand". In: Thammasat Universität, Faculty of Liberal Arts, German Department (ed.): Dokumentation der Tagungsbeiträge. IV. Thailändisches Germanistentreffen, 22.-24. März. Bangkok: 46-52.

Bisang, Walter (1992): Das Verb im Chinesischen, Hmong, Vietnamesischen, Thai und Khmer: vergleichende Grammatik im Rahmen der Verbserialisierung, der Grammatikalisierung und der Attraktorpositionen. Tübingen.

Ganeshan, Vridhagiri (2000): "Hermann Hesses Gedicht 'Bhagavad Gita' - eine Interpretation aus interkultureller Sicht". TDLV-Forum 5: 1-6.

Granger, Syviane/Lerot, Jacques/Petch-Tyson, Stephanie (eds.) (2003): Corpus-based Approaches to Contrastive Linguistics and Translation Studies. Amsterdam/New York.

Johansson, Stig (2003): "Contrastive linguistics and corpora". In: Granger, Syviane/Lerot, Jacques/Petch-Tyson, Stephanie (eds.): Corpus-based Approaches to Contrastive Linguistics and Translation Studies. Amsterdam/New York: 31-44. 
Kaneko, Tohru (2001): "Kontrastive Analysen Deutsch-Japanisch: eine Übersicht". In: Helbig, Gerhard et al. (eds.): Deutsch als Fremdsprache. Ein internationales Handbuch. Berlin/New York: 451-457.

Keul, Norman C. (1981): "A Selected, Annotated Bibliography of English-German Contrastive Linguistics". Die Unterrichtspraxis/Teaching German 14/1: 105-116.

König, Ekkerhard (2001): "Kontrastive Analysen Deutsch-Englisch: eine Übersicht". In: Helbig, Gerhard et al. (eds.): Deutsch als Fremdsprache. Ein internationales Handbuch. Berlin/New York: 324-330.

Kelz, Heinrich P./Kummer, Manfred (1989): Beiträge zur Phonetik des Thailändischen. Hamburg.

Krumm, Hans-Jürgen (2003): "Deutsch von außen - in der Inlandsgermanistik". In: Stickel, Gerhard (ed.): Deutsch von außen. Berlin/New York: 259-273. (= Jahrbuch des Instituts für Deutsche Sprache 2002).

Kummer, Manfred (1990): "Höflichkeit in Südostasien. Verhaltensmuster und Sprachgebrauch". In: Südostasien-Institut e.V. (ed): Brücken nach Südostasien. Beiträge zum Interkulturellen Verstehen. Heildelberg: 35-46.

Kummer, Manfred (2006): "Politeness in Thai". In: Watts, Richard J. (ed.): Politeness in Language. Berlin/New York: 325-336.

Lepper, Marcel (2008): "Welche Auslandsgermanistik?" Jahrbuch der Deutschen Schillergesellschaft 52: 14-17.

Noochoochai, Ponlasit (1979): Temporal Aspect in Thai and English: A Contrastive Analysis. London.

Otrakul, Ampha et al. (1982): Thai-Deutsches Wörterbuch. Bangkok.

Saengaramruang, Wanna (1986-1991): Waiyakornjeraman Lem 1-3 ['Deutsche Grammatik Band 1-3']. Bangkok.

Saengaramruang, Wanna (2007): "Deutschunterricht und Germanistikstudium in Thailand: früher, heute und morgen". INFO DaF 34/4: 341-372.

Sookgasem, Prapa (1990): Morphology, Syntax and Semantics of Auxiliaries in Thai. PhD Thesis, University of Arizona. Tucson.

Taatloha, Prisna et al. (1989-1992): Viel Spaß mit Deutsch. Ein Lehrwerk für Jugendliche in Thailand. Band 1-3. Bangkok.

Velder, Christian (1998): "Beiträge deutscher Wissenschaftler zur Thailandkunde". TDLVForum 3/98: 8-13.

Wattanaguhn, Pornsan/Banjongthad, Wanpen/Paripanyaporn, Kalaya (1988): Lesen macht Spaß. Bangkok.

Wattananguhn, Pornsan (2008): "Germanistik und Deutschunterricht in Thailand definieren sich neu". In: Thammasat Universität, Faculty of Liberal Arts, German Department (ed.): Dokumentation der Tagungsbeiträge. IV. Thailändisches Germanistentreffen, 22.-24. März. Bangkok: 34-45.

Zifonun, Gisela (2001): Zur Grammatik des Deutschen im europäischen Vergleich: Das Pronomen. Teil 1: Überblick und Personalpronomen. Mannheim.

Zifonun, Gisela (2007): Zur Grammatik des Deutschen im europäischen Vergleich. Das Pronomen. Teil IV: Indefinita im weiteren Sinne. Mannheim.

\section{Appendix: Liste der thai-deutschen linguistischen kontrastiven Studien}

Akkramas, Pakini/Traoré, Salifou (2002): "Ein Diskussionsbeitrag zu Zeit und Tempus aus kontrastiver Sicht (Deutsch-Thai)". Die Brücke. Zeitschrift für Germanistik in Südostasien 1: $1-10$.

Attaviriyanupap, Korakoch (2006): "Ausspracheabweichungen im Hochdeutsch thailändischer Immigrantinnen in der Deutschschweiz". Linguistik online 1/26: 15-30. www.linguistik-online.de/26_06/attaviriyanupap.html. 
Attaviriyanupap, Korakoch (2007): ich mut geles gelies gelies wat lesen. Der Hochdeutscherwerb durch thailändische Immigrantinnen in der Schweiz. Dissertation Universität Bern.

Attaviriyanupap, Korakoch (2008): "Thailändisches Deutsch? Ausspracheabweichungen bei thailändischen Immigratinnen in der Deutschschweiz". In: Thammasat Universität, Faculty of Liberal Arts, German Department (ed.): Dokumentation der Tagungsbeiträge. IV. Thailändisches Germanistentreffen, 22.-24. März. Bangkok: 184-199.

Benjawattananan, Thanarat (2006): Die es-Konstruktionen im Deutschen: ihre Funktion und ihre Übertragung ins Thailändische. MA-Arbeit. Chulalongkorn Universität.

Benjawatananun, Thanarat/Saengaramruang, Wanna (2007): "Die es-Konstuktionen im Deutschen: ihre Funktion und ihre Übertragung ins Thailändische". TDLV-Forum 11: 15-40.

Kaewwipat, Noraseth (2007): Kontrastive Lesegrammatik Deutsch - Thai für den Unterricht Deutsch als Fremdsrpache in Thailand - Untersuchungen am Beispiel des Nominalstils. Kassel.

Laisanitsereekul, Unyamanee (2003): Verbvalenz und Satzstruktur: Eine kontrastive Analyse Deutsch-Thai. MA-Arbeit. Ramkhamhaeng Universität.

Laisanitseereekul, Anyamanee (2003): "Satzstruktur im Deutschen und im Thai: Gemeinsamkeiten und Unterschiede". Die Brücke. Zeitschrift für Germanistik in Südostasien 3: 14-19.

Littmann, Michael (1988-1999): "Thai and German - two different worlds? Relationship between the Thai and the German language". Humanities and Social Science. Journal of Khon Kaen University 2/10: 10-12.

Loto, Praiwan (2006): Höflichkeit. Eine Untersuchung zur sprachspezifischen Binnengliederung eines Begriffs (Deutsch-Thailändisch). MA-Arbeit. Ramkhamhaeng Universität.

Namsoongnein, Chutamat (1987): Entsprechungen der deutschen Modalpartikeln im Thai. Eine kontrastive Untersuchung: denn, doch, mal. MA-Arbeit. Universität Konstanz.

Ngaosuwan, Narümon (1978): Hauptschwierigkeiten der thailändischen Studenten beim Erlernen der Zielsprache Deutsch. MA-Arbeit. Chulalongkorn Universität.

Palabhut, Tantip (2001): Sprache der Werbung: Eine kontrastive Untersuchung zur stilistischrhetorischen Gestaltung von Werbeslogans im Deutschen und im Thailändischen. MAArbeit. Chulalongkorn Universität.

Ralugmool, Wilairat (2002): Die Negation im Deutschen und im Thai. MA-Arbeit. Chulalongkorn Universität.

Saengaramruang, Wanna (2000): Kan Plae Kham Abtönungspartikel Nai Phasa Jöraman Pen Phasathai ['Die Übersetzung der deutschen Abtönungspartikeln ins Thailändische']. Journal of Faculty of Arts 1/29: 161-190.

Saengaramruang, Wanna (2001): "Die Übersetzung der deutschen Abtönungspartikeln in der Literatur ins Thailändische". TDLV-Forum 6: 49-66.

Saengaramruang, Wanna (2002): Abtönungspartikel Nai Phasa Jöraman Lae Chetanarom Nai Kan Suesan ['Abtönungspartikeln im Deutschen und ihre Kommunikationszwecke']. Bangkok.

Saengaramruang, Wanna (2003): "Thai-Deutsches Wörterbuch für thailändische Deutschlernende: Spezifische Merkmale der beiden Sprachen". In: Ramkhamhaeng Universität, Faculty of Fine and Applied Arts, Department of Western Languages (ed.): Tagungsband. 2. Thailändisches Germanistentreffen. 7.-8. Oktober 2003. Bangkok: 169-191.

Saengaramruang, Wanna (2008): Prayokkam Phasa Jöraman. Kan Thay Khwam Pen Phasa Thai ['Passivformen im Deutschen: Ihre Übertragung ins Thailändische']. Bangkok.

Sriuranpong, Wilita (1997a): Wortstellung im Deutschen und im Thai. Heidelberg.

Sriuranpong, Wilita (1997b): "Die Objektstellung im Deutschen und im Thai". TDLVForum 2: 15-23.

Sriuranpong, Wilita (2002): "Internationalismen im Deutschen und im Thai". TDLV-Forum 7: $222-238$. 
Sriuranpong, Wilita (2007): "Die Merkmale des Passivs im Deutschen und im Thai. Eine kontrastive Untersuchung unter besonderer Berücksichtigung seiner Funktionen". TDLVForum 11: 41-51.

Sriuranpong, Wilita (2008): "Die Merkmale des Passivs im Deutschen und im Thai. Eine kontrastive Untersuchung unter besonderer Berücksichtigung seiner Funktionen". In: Thammasat Universität, Faculty of Liberal Arts, German Department (ed.): Dokumentation der Tagungsbeiträge. IV. Thailändisches Germanistentreffen, 22.-24. März. Bangkok: 173-183.

Suriyajun, Chanokpim (2003): Nominalkomposita im Deutschen und im Thai. MA-Arbeit. Chulalongkorn Universität.

Traoré, Salifou (2008): Interkulturelle Germanistik. Konzeptionelle Überlegungen zu einer Grammatik aus eigener und fremder Perspektive im Deutschen als Fremdsprache. Frankfurt am Main etc.

Voraurai, Sornprahm (2002): Die Relativsätze im Deutschen und im Thai. MA-Arbeit. Chulalongkorn Universität.

Woraurai, Sornpram (2003): "Die Relativätze im Deutschen und im Thai". TDLV-Forum 8: $76-87$. 